\title{
MÁS ALLÁ DEL RELOJ COMO MODELO DEL SER VIVO: LA DISTINCIÓN MÁQUINA NATURAL Y MÁQUINA ARTIFICIAL EN LEIBNIZ*
}

\author{
Ronald Durán Allimant** \\ http://orcid.org/0000-0001-8447-4446 \\ ronald.duran@upla.cl
}

RESUMEN Durante el siglo XVII, el reloj parece el modelo más adecuado para pensar los seres vivos. El filósofo alemán G. W. Leibniz (1646-1716) es parte de la tradición mecanicista que concibe los seres vivos a partir del modelo del reloj o de los autómatas, pero establece una distinción esencial entre máquinas naturales y artificiales, que muestra los limites de este modelo. Las primeras son máquinas infinitamente complejas, máquinas dentro de máquinas ad infinitum, las segundas no, alcanzan un límite de complejidad. Esta distinción obliga a ir más allá del reloj como modelo de los seres vivos, pues este modelo resulta insuficiente para comprender la dinámica propia de los seres vivos, en al menos dos aspectos: a) deja sin explicar el origen de la estructura oforma del ser vivo; b) no establece un principio interno de actividad que fundamente la unidad dinámica y estructural del ser vivo. Con su noción de organismo o máquina natural, Leibniz intenta resolver estas insuficiencias del modelo puramente mecánico: a) en su propuesta el ser vivo no se constituye de manera mecánica o serial, sino de una vez, por un acto de creación; b) la conservación del ser vivo en el tiempo sólo es comprensible a partir de un principio de actividad intrínseca que dota de unidad, actividad y estructura al viviente.

* Artigo submetido em 11/06/18 e aprovado em 08/10/18. La presente investigación ha sido posible gracias al financiamiento otorgado por el proyecto FONDECYT Postdoctoral № 3160241 de CONICYT, del cual el autor del presente escrito es el investigador responsable.

** Universidad de Playa Ancha (UPLA). Valparaíso, Chile. 
Palabras clave Leibniz, máquina natural, ser vivo, organismo, máquina artificial, reloj.

ABSTRACT During the seventeenth century, the clock seems the most appropriate model for thinking about living beings. The German philosopher G. W. Leibniz (1646-1716), is part of the mechanical tradition that conceives living beings from the clock or the automata's model, but he establishes an essential distinction between natural and artificial machines. This distinction shows the limits of the mechanical model. The machines of nature are infinitely complex machines, machines within machines ad infinitum; the artificial machines, instead, reach a limit of complexity. This distinction forces us to go beyond the clock as a model of living beings, because this model is insufficient to understand the dynamics of living beings, in at least two aspects: a) it is not able to explain the origin of the living being's structure or form; b) it does not establish an internal principle of activity grounding the living being's dynamic and structural unit. With his notion of organism or natural machine, Leibniz tries to solve these insufficiencies of the purely mechanical model: a) in his proposal, the living being is not constituted mechanically or serially, but at once, by an act of creation; b) the conservation of the living being in time is only understandable from a principle of intrinsic activity that provides unity, activity and structure to living beings.

Keywords Leibniz, natural machine, living being, organism, artificial machine, clock.

\section{Introducción: el reloj como modelo del ser vivo en el mecanicismo del siglo XVII}

Los siglos XVI y XVII muestran una abundancia de autómatas, de máquinas automáticas, en iglesias, teatros, palacios, jardines. Proliferan relojes mecánicos, molinos, figuras humanas y animales con automovimiento, teatros mecánicos en cuevas (grottos), juegos de agua, entre otros (Montaigne, 1889, p. 323; Riskin, 2016p. 30). A pesar de sus diferencias, estos artilugios mecánicos obedecían a un mismo principio: diversas partes o engranajes en funcionamiento mecánico coordinado. Estos autómatas, a diferencia de los instrumentos o herramientas manuales como palas o martillos, poseían una cierta autonomía funcional; pues la acción que los ponía en movimiento no determinaba la forma de los movimientos coordinados que tenían lugar en la máquina, su funcionamiento. 
De todos los ejemplos de autómata, el reloj mecánico es sin duda el mecanismo ejemplar, el paradigma: «En los siglos XVII y XVIII, la metáfora del reloj pasó a ser muy frecuente, más, con toda probabilidad, que ninguna otra» (Mayr, 2012, p. 47). El reloj pasó a convertirse en la metáfora o modelo clave en la comprensión de los seres vivos y los cuerpos orgánicos, en la concepción del mundo como un gran mecanismo de relojería, y de Dios como relojero que ha diseñado este mundo (Rossi, 1966, p. 131). El funcionamiento de los autómatas y la coordinación de sus partes en movimiento parecían desvelar el funcionamiento secreto de la naturaleza.

La comprensión mecánica de los seres vivos implica un doble movimiento: «Por una parte, la identificación de las funciones del viviente con el efecto de movimientos que siguen leyes que son comunes a todos los cuerpos, inanimados y animados; por otra parte, la concepción del cuerpo humano como un cuerpo compuesto de partes diversamente movidas y ordenadas cuyo conjunto es causa de efectos subordinados al cumplimiento de un fin» (Andrault, 2016, p. 10) ${ }^{1}$. La comprensión mecánica de los seres vivos elimina la frontera que existía entre el mundo de los cuerpos animados e inanimados, quedando inscritos los seres vivos en el mundo físico, entendido en sentido puramente mecánico (figura, movimiento, magnitud) (AT VII: 42; 2002, p. 73). Por otra parte, los autómatas conjugan dos aspectos que serán esenciales en el enfoque epistemológico de la época: primero, la posibilidad de analizar, de separar en partes discretas, de reconducir el funcionamiento global de una máquina a sus partes elementales, $\mathrm{y}$, en segundo lugar, una suerte de síntesis, la coordinación de estas partes que da lugar a un funcionamiento de conjunto que aúna movimiento serial y coordinación global.

Todas estas ideas quedan de manifiesto de manera ejemplar en la concepción de cuerpo orgánico de Descartes. En su Tratado del hombre, afirma:

\footnotetext{
Supongo que el cuerpo no es otra cosa que una estatua o máquina de tierra a la que Dios da forma $[\ldots y]$ también dispone en su interior todas las piezas requeridas para lograr que se mueva, coma, respire y, en resumen, imite todas las funciones que nos son propias [...] Conocemos relojes, fuentes artificiales, molinos y otras máquinas similares que, habiendo sido realizadas por el hombre, sin embargo poseen fuerza para moverse de modos diversos en virtud de sus propios medios [...] No me detendré en realizar una descripción de los huesos, los nervios, los músculos, las arterias, el estómago, el hígado, el bazo, el corazón, el cerebro ni de todas las otras piezas de que está formada [esta máquina] (AT XI: 120; 1980, p. 50). ${ }^{2}$
}

1 La traducción es nuestra. En lo que sigue hemos usado las traducciones publicadas cuando están disponibles, en caso contrario hemos traducido directamente del idioma original.

2 En La description du corps humain (1648), Descartes afirma en relación a su análisis del cuerpo: «[...] que se tenga primero una concepción general de toda la máquina que describiré» (AT XI: 226). Luego describe 
Para Descartes los cuerpos orgánicos no son más que máquinas, ciertamente más sutiles que las hechas por el ser humano, en tanto creaciones divinas o naturales, pero máquinas igualmente. Tal como afirma: «[...] no reconozco diferencia alguna entre las máquinas que construyen los artesanos y los cuerpos que la naturaleza por si misma ha formado» (AT IX: 321; 2002p. 410). Se trata aquí de una diferencia de grado y no de naturaleza.

[...] sabiendo cuántos y cuán distintos autómatas o máquinas movientes puede construir la industria humana, sin emplear en ellos más que un número de piezas muy pequeño, en comparación con la gran multitud de huesos, músculos, nervios, arterias, venas y demás partes que hay en el cuerpo de cada animal, podrán considerar este cuerpo como una máquina que, habiendo sido hecha por las manos de Dios, está incomparablemente mejor ordenada y es capaz de movimientos más admirables que ninguna de las que pueden ser inventadas por el hombre (AT VI: 55; 2004, p. 95).

De esta manera, la diferencia entre las máquinas artificiales y las que podríamos llamar «máquinas naturales», sólo es una diferencia de grado y no de esencia, dependiente de la cantidad y sutileza de las piezas que las constituyen, lo que implica a su vez diferencias en el ordenamiento y funcionamiento. En sentido estricto, la distinción entre lo natural y lo artificial se anula en Descartes, de tal manera que las máquinas pueden llamarse naturales o artificiales con igual propiedad. Afirma Descartes: «las reglas de la mecánica pertenecen a la Física, de suerte que todos los seres construidos mediante artificio son, de acuerdo con tales reglas, naturales» (AT IX: 321; 2002, p. 410). Y estas máquinas pueden ser comprendidas desde una perspectiva puramente material, excluyendo las formas aristotélicas, y concibiendo como esencia de la materia la sola extensión. Así, la mera disposición de los órganos puede explicar los movimientos no voluntarios, sin necesidad de recurrir al alma, al igual que «no tenemos que juzgar que hay un alma en un reloj, que hace que él muestre las horas» ( $L a$ description du corps humain, AT XI: 226. Geometrizando la materia, Descartes espera entender el funcionamiento de lo vivo partiendo solo de la consideración de la figura, el tamaño y el movimiento. En este contexto mecanicista, el alma humana es situada como una excepción, que permite salvar su inmortalidad; sin embargo, los animales, al carecer de alma, son considerados puramente maquinales: «[...] si hubiese máquinas tales que tuvieran los órganos y la figura de un mono, o de cualquier otro animal desprovisto de razón, no tendríamos 
medio alguno de conocer que no eran, en todo, de la misma naturaleza que esos animales» (AT VI: 56; 2004, p. 97; Des Chene, 2001, p. 108).

Así, durante el siglo XVII, con la consolidación del paradigma mecanicista, el reloj, como ejemplo por excelencia de máquina automática, constituyó un modelo preferente para considerar lo vivo y su funcionamiento, y sirvió para difuminar la frontera entre lo artificial y lo natural. Tal como afirma Descartes: «Cuando un reloj marca las horas por medio de las ruedas de las que está formado, no es tal efecto menos natural de lo que es que un árbol produzca frutos» (AT IX: 321-322; 2002, p. 410) ${ }^{3}$.

\section{Insuficiencias del reloj como modelo del ser vivo: el problema del origen de las formas biológicas y el problema de su conservación en el tiempo}

En el contexto mecanicista, la generación y la destrucción de organismos sólo pueden comprenderse como agregación y desagregación de partes, respectivamente, y el movimiento sólo puede ser fruto de choques, proveniente en última instancia de fuentes externas a los propios sistemas mecánicos. La explicación mecánica deja de lado las formas y con esto el principio que servía a Aristóteles para diferenciar entre lo natural y lo artificial, a saber, la localización de la forma que constituye el principio de movimiento. Para lo natural, como los seres vivos, el principio de movimiento es interno, mientras que para lo artificial es externo, yace en otro (Física II: 192b1-24). En el paradigma mecánico, por su parte, lo natural y lo artificial, los seres vivos y los autómatas mecánicos son igualmente máquinas, como ya hemos visto. La forma, lo que hoy podríamos llamar organización, queda ausente en el modelo de explicación mecanicista (Des Chene, 1996, p. 342). Veamos algunas de las insuficiencias principales de este esquema explicativo mecanicista, que tiene al reloj como modelo ejemplar.

Una primera insuficiencia se refiere al origen de las formas biológicas, al origen de la organización biológica. La explicación del surgimiento de lo vivo por medio del mero choque de masas en movimiento resulta inverosímil. La constitución de un reloj funcional a partir de la mera combinación aleatoria de sus componentes, sería poco menos que milagrosa. Aunque los componentes de un reloj indican en cierta medida en su propia figura la función o ubicación

3 En la edición en francés: «Et il est certain que toutes les règles des Méchaniques appartiennent à la Physique.... en sorte que toutes les choses qui sont artificielles, sont avec cela naturelles. Car, par exemple, lorsqu'une montre marque les heures par le moyen des roues dont elle est faite, cela ne lui est pas moins naturel qu'il est à un arbre... de produire ses fruits» (AT IX: 321-322). Mientras que en la versión latina (anterior a la edición francesa) tenemos: «nec minus naturale est horologio, ex his vel illis rotis composito, ut horas indicet, quam arbori ex hoc vel illo femine ortae, ut tales fructus producat» (AT VIII-I: 326). 
en la estructura de un reloj, esta indicación no es suficientemente definida como para acotar de manera absoluta las posibles combinaciones o articulaciones; existe una subdeterminación de los componentes respecto de la organización de la que forman parte. Más aún, las propias partes componentes de un mecanismo sólo pueden ser tales si ya se tiene de antemano un diseño a partir del cual son consideradas, diseño que se insinúa en la figura de cada una de ellas. El diseño preexiste a la articulación de las partes. La sola consideración de las partes de un sistema mecánico no permite dar cuenta de la forma o diseño que configura un mecanismo funcional. De esta manera, el reloj no puede dar cuenta de su propio diseño, necesita de un diseñador. La aplicación de este esquema al ser vivo, obliga así a incorporar un elemento externo al propio organismo, un diseñador (Dios, en el contexto de la época moderna), que establece el plan funcional y pone en movimiento el sistema mecánico. ${ }^{4}$

Una segunda insuficiencia del reloj como modelo del ser vivo, se refiere a la actividad propia de la que está dotada un ser vivo y al mantenimiento de esta actividad en el tiempo, lo que hoy denominamos la capacidad de equilibrio dinámico de los seres vivos o estabilidad. ¿Cómo explicar esta característica de los seres vivos mecánicamente, es decir, apelando sólo a la figura, el tamaño y el movimiento? ¿Cómo explicar con estos medios la conservación en el tiempo de la organización de un ser vivo y su diferenciación respecto a un medio (que implica tanto una frontera espacial como funcional) $?^{5}$ ¿Qué hace que un organismo siga siendo uno y el mismo, sin ser sin embargo lo mismo, es decir modificando constantemente sus partes componentes? Si desde la perspectiva mecánica el surgimiento de una organización diferenciada resulta milagroso, perpetuar esta organización en el tiempo y en el espacio implica repetir este milagro a cada instante.

Estos dos problemas principales del mecanicismo, el surgimiento de la organización funcional del ser vivo, y su conservación en el tiempo y en el espacio, fueron enfrentados por Leibniz con su propuesta del ser vivo como organismo o substancia corpórea, y su concepción del cuerpo orgánico como máquina de la naturaleza.

4 Lo que sirve para defender el argumento del diseño para demostrar la existencia de Dios. Cf. Mayr, 2012, p. 111.

5 La configuración de la unidad propia de los seres vivos implica que se establece una suerte de clausura funcional (una diferencia entre un adentro y un afuera funcional) y una clausura espacial (una frontera espacial). Cf. Maturana y Varela, 1994, p. 65. 


\section{El modelo de ser vivo en Leibniz: el organismo como unidad dinámica y estructural.}

Leibniz está consciente de las insuficiencias del modelo puramente mecanicista, e intenta remediarlas extendiéndolo y complementándolo. Pone de relieve que tratar de explicar los seres vivos apelando a la pura extensión resulta imposible, ya que la mera extensión, divisible al infinito, vuelve imposible la existencia de unidades verdaderas, lo que implicaría la no existencia de seres reales, pues para él ser es sinónimo de unidad (AA VI: 583; Jalabert, 1947; Woolhouse, 1993). Si los seres vivos no constituyen unidades verdaderas, serían meros fenómenos bien fundados o en el peor de los casos meros agregados, sin unidad substancial, pura unidad puesta por quien percibe. Así pues, los cuerpos orgánicos no pueden ser comprendidos sólo desde la noción de extensión porque la extensión implica una división infinita, no sólo potencial sino actual. Leibniz considera que para ser substancias en sentido estricto, los seres vivos han de estar dotados de una actividad intrínseca, espontánea, deben estar dotados de un principio de actividad intrínseco que establezca la unidad dinámica y estructural del ser vivo (GP IV, 472; WF 21; Durán, 2016). Este principio es referido de diversas formas por Leibniz a lo largo de su obra, a saber: forma substancial, fuerza, entelequia o mónada (sustancia simple, sin partes, espontánea, que no interactúa causalmente, dotada de percepción y apetito) (AA VI, 4B: 1542, 1583; OFC 2: 170, 200). Hay que complementar lo material incorporando una dimensión formal o mental en lo material.

La descripción más detallada, pero al mismo tiempo problemática, de la estructura de los seres vivos es planteada por Leibniz en su carta a De Volder del 20 junio de 1703. En ella describe al animal o ser vivo, de la siguiente manera:

(1) entelequia primitiva o alma; (2) materia prima o potencia pasiva primitiva; (3) mónada completa formada por estas dos; (4) masa o materia secunda, esto es, máquina orgánica, a la que concurren innumerables mónadas subordinadas; (5) animal o substancia corpórea, a la que la mónada dominante da unidad dentro de la máquina (GP II, 252; OFC 16B, 1200).

Los cinco elementos que constituyen el animal o la substancia corpórea se presentan aquí de manera estructural, sincrónica o estática. Sin embargo, para Leibniz, la unidad, y con ello la substancialidad, es concebible sólo como intrínsecamente activa, como ya dijimos. Además, la unidad del ser vivo en Leibniz no es simple sino infinitamente compleja. Esto porque la mónada que dota de unidad a un ser vivo domina (organiza o coordina) un conjunto infinito de mónadas ordenadas en una estructura anidada, donde cada una de ellas domina a su vez a otro conjunto de mónadas al infinito. El ser vivo tiene así 
una estructura jerárquica infinita. El conjunto de mónadas dominadas constituye el cuerpo orgánico del ser vivo, o la «máquina natural», máquina que es tal no sólo en el conjunto sino en cada una de sus partes hasta el infinito. El ser vivo en su conjunto es lo que Leibniz denomina una substancia corpórea (GP II, 252; OFC 16B; Lodge, p. 264; Phemister, 2005, p. 49). ${ }^{6}$

En la descripción de la carta a De Volder, se plantean algunos puntos principales que permiten entender la relación entre actividad y unidad en el ser vivo, tal como lo concibe Leibniz, a saber: a) la relación entre actividad espontánea y unidad, b) la relación jerárquica dentro del animal o ser vivo, c) la estructuración al infinito de esta unidad jerárquica, d) la relación que puede establecerse entre clausura de dominio (o clausura funcional) y clausura espacial. El primer punto se explicita al indicar la necesidad de una mónada dominante que con su actividad espontánea y unidad vuelve uno al animal o ser vivo. Se menciona explícitamente la entelequia o principio de actividad. La unidad del ser vivo o substancia corpórea, requiere, por tanto, una dinámica propia. Ahora bien, aunque aquí no se menciona explícitamente, la actividad de la mónada dominante no es la única requerida, sino que la actividad de las mónadas que componen el cuerpo orgánico, las mónadas subordinadas, es fundamental también (Durán, 2017, p. 87). Otro punto destacado, también usando como base la noción de dominación o dominio, es que esta relación establece una jerarquía: mónada dominante - mónada dominada o subordinada. No todas las mónadas juegan el mismo rol dentro del ser vivo o substancia corpórea, sino que unas están supeditadas a otras, estructura que se continúa al infinito. «La ligazón y el orden de las cosas hace que el cuerpo de todo animal y de toda planta esté compuesto de otros animales y de otras plantas, o de otros seres vivos y orgánicos; y que, por consiguiente, haya subordinación, y que un cuerpo, una sustancia, sirva a otra; por tanto, su perfección no puede ser la misma» (GP VI: 245; OFC 10: 238). Aquí tenemos el tercer punto que hay que considerar. Cada mónada subordinada es, a su vez, mónada dominante respecto de aquellas que configuran su propio cuerpo orgánico. Y esto, como se ha dicho, hasta el infinito (se puede pensar aquí en una suerte de estructura fractal autorreplicante). Esta estructura jerárquica al infinito, sirve a Leibniz para distinguir las máquinas naturales, los seres vivos, de las máquinas artificiales, como veremos luego. Ahora bien, un último punto que se esboza en esta descripción del ser vivo, es la relación entre lo que podemos llamar clausura de dominio (o clausura funcional), establecida por las relaciones entre las mónadas que constituyen el 
ser vivo, y la clausura espacial, que permite distinguir como unidad espaciotemporal un animal o ser vivo. En Leibniz estas dos clausuras no coinciden porque la coherencia y unidad dinámica y estructural del ser vivo va más allá de la unidad o coherencia como unidad espacial. Es a esta unidad o clausura funcional que apunta Leibniz cuando habla de «organismo».

Leibniz, sin ser el primero en usar el término «organismo», contribuye, sin embargo, a su difusión (Duchesneau, 2010, p. 98). Lo comprende en un sentido distinto al que es común hoy en día. En la biología contemporánea, el término «organismo» se usa en referencia a un ser vivo singular considerado como una entidad viva individual, sobre todo desde el punto de vista espacial. Sin embargo, en Leibniz «organismo» se refiere más bien a la organización del animal, a su unidad estructural y dinámica, tal como lo indica en su carta a Lady Masham del 30 de junio de 1704: «[...] organismo, es decir, orden y diseño [ingenuity]» (GP III: 356; Duchesneau, 2010, p. 85). El término «organismo» apunta al mecanismo y las relaciones estructurales y dinámicas que permiten que el animal sea el mismo a través de sus diferentes cambios. De esta manera, al hablar de organismo Leibniz apunta a la relación entre mónadas dominantes y subordinadas, relación que persiste a través de los cambios o transformaciones del ser vivo.

Conviene aclarar aquí un uso en cierta medida ambiguo de los términos que refieren al ser vivo en Leibniz. Como aparece en la descripción de la carta a De Volder, el ser vivo o animal equivale a la substancia corpórea, es decir, a la unidad de mónada dominante y mónadas dominadas, éstas a su vez constituyen el cuerpo orgánico. En ocasiones Leibniz hace equivalentes cuerpo orgánico y máquina de la naturaleza, pero en ocasiones se refiere al ser vivo como máquina de la naturaleza. ${ }^{7}$ Aquí consideraremos como sinónimos estos términos a no ser que hagamos la diferencia de manera explícita, en tanto cada uno de ellos apunta a aspectos complementarios del ser vivo como unidad dinámica y estructural.

\section{La diferencia esencial entre máquina natural y máquina artificial} en Leibniz: el ser vivo como máquina infinitamente compleja

Aunque Leibniz sigue comprendiendo el ser vivo desde la perspectiva del mecanicismo como máquina, no considera, a diferencia de Descartes, que el

7 Como ejemplo del primer caso tenemos: «Ninguna masa es tan carente de forma o tan pequeña que no contenga en ella algún cuerpo orgánico o máquina de la naturaleza [corpus organicum, seu machinam naturae]» (Leibniz, 2016, p. 249). Como ejemplo del segundo caso, tenemos: "Así a partir del paralelismo entre causas material y formal en los seres vivos, o en las máquinas de la naturaleza [in vivientibus, seu in naturae Machinis], somos conducidos al paralelismo entre causas eficientes y fines» (Leibniz, 2016, p. 23). 
reloj o las máquinas artificiales sean idénticos a los seres vivos, y por lo tanto cuestiona que puedan servir de modelo adecuado para entender su naturaleza propia. Esto queda de manifiesto en la distinción radical que establece entre máquinas naturales y máquinas artificiales, tanto desde una perspectiva estructural como dinámica.

Para Descartes, como ya vimos, la diferencia entre máquinas naturales y máquinas artificiales era sólo una diferencia de grado, dependiente de la cantidad de las partes y la sutileza de la construcción. Por el contrario, para Leibniz la diferencia es radical, esencial, «[...] dans le genre même» (GP IV: 482; WF: 16; GP VI: 618; Smith, 2011, p. 97; Smith y Nachtomy, 2011, p. 61; Fichant, 2003; Duchesneau, 2010, p. 85). Para él, las máquinas naturales, los seres vivos, son máquinas infinitamente complejas, máquinas dentro de máquinas ad infinitum, mientras que las máquinas artificiales no lo son, pues alcanzan un límite de complejidad. El autómata o máquina de la naturaleza «es máquina no sólo en el todo, sino incluso en las partes más pequeñas que quepa notar» (GP VI: 599; OFC 2: 344). La complejidad infinita de las máquinas naturales, no es sólo una cuestión del número infinito de partes, no es sólo una cuestión estructural, sino al mismo tiempo funcional o dinámica; pues los seres vivos funcionan como un todo orgánico, algo que las máquinas artificiales no pueden hacer. Como hemos visto, el ser vivo para Leibniz es una unidad organizacional (dinámica y estructural) que denomina «organismo», y que podemos considerar opuesta al mero mecanismo. Un autómata mecánico, como el reloj, no está dotado de unidad orgánica como la del ser vivo (máquina divina o autómata natural), no posee unidad real: «A mi modo de ver, la unidad de un reloj [...] es completamente diferente de la de un animal; pues un animal puede ser una substancia con una genuina unidad, como lo que llamamos "yo" en nosotros mismos, mientras que un reloj es nada más que un agregado» (GP VI: 618;WF 48). O bien: «Una verdadera unidad [...] no podría suceder ni en las máquinas artificiales ni en la simple masa de la materia por muy organizada que pudiera estar; a ésta sólo la podemos considerar como un ejército o un rebaño, o como un estanque lleno de peces, o como un reloj compuesto de resortes y de ruedas» (GP IV: 482; OFC: 245). De alguna manera, en cada una de las partes de un ser vivo está el todo o la referencia al todo como unidad fundamental, y este todo implica, a su vez, la referencia a cada una de las infinitas partes. Una máquina de la naturaleza o ser vivo posee una estructura dinámica y jerárquica en la que cada máquina componente tiene un lugar funcional en relación al ser vivo considerado como un todo (que a su vez es distinto de su medio). Una máquina de la naturaleza que careciera de unidad propia (que careciera de una mónada dominante) estaría en la misma situación que un reloj, pues cada una de sus partes, cada una de 
las máquinas o animales que lo constituyen, no tendría una relación funcional definida respecto al funcionamiento del cuerpo como un todo. Sólo tendrían, quizás, una relación estructural, pero no jerárquica ni dinámica. Por esta razón, resulta importante destacar que la distinción entre máquina artificial y máquina natural que plantea Leibniz, no es sólo una diferencia estructural, sino también una diferencia dinámica o funcional, pues en sentido estricto un reloj también podría ser considerado estructuralmente infinito, pues cada una de sus partes componentes está constituida por seres vivos o animales, es decir por máquinas, hasta el infinito (al igual que todo lo material, según Leibniz).

Cabe mencionar que la distinción entre máquina artificial y máquina natural en Leibniz, apelando a la articulación infinita de sus partes, fue fuertemente influida, aunque no completamente configurada, por el perfeccionamiento del microscopio y el descubrimiento de seres animados en partes que antes parecían elementos últimos e inanimados («infinidad de animales en la más pequeña gota de agua», AA II-2: 254), como muestra por ejemplo el trabajo del microscopista holandés Anton van Leeuwenhoek (Smith, 2011, p. 178; Duchesneau, 2010, p. 91; Wilson, 1995, p. 176). El maravilloso mundo microscópico parecía confirmar que la vida sólo nace de la vida, que las formas no surgen de manera espontánea de la materia, sino que surgen de semillas microscópicas, de organismos preformados, como veremos más adelante (GP VI: 553).

Por último, tengamos en cuenta que Leibniz retoma en cierta medida la distinción aristotélica entre lo natural y lo artificial, no sólo al reincorporar las formas substanciales, sino al establecer que el desarrollo de las máquinas naturales debe ser intrínseco, mientras que el de las máquinas artificiales no (al menos como unidad real). Las máquinas naturales, los seres vivos, siguen principios de construcción distintos de las máquinas artificiales, en tanto actualizan directamente el plan de la naturaleza, no recibiendo una forma externa, sino transformándose a partir de una preforma (GP VI: 619; 544; Duchesneau, 1998, p. 324).

Aunque Leibniz sigue concibiendo los seres vivos a partir del paradigma mecanicista como máquinas, la frontera radical entre máquinas artificiales y máquinas naturales enfatiza los límites del mero mecanismo y del modelo del reloj como explicación de los seres vivos.

\section{La propuesta de superación del modelo del reloj a partir del ser vivo como organismo}

Con su propuesta del ser vivo concebido como una unidad dinámica y estructural (organismo o substancia corpórea), que posee un cuerpo orgánico 
o máquina de la naturaleza, Leibniz intenta superar las dificultades del modelo puramente mecánico del ser vivo basado en el reloj.

La primera insuficiencia del mecanicismo se refería al origen del ser vivo y su organización funcional. La materia concebida como mera extensión, como en la filosofía cartesiana, implica una división al infinito y la carencia de un principio intrínseco de actividad, de tal manera, que por sí sola no puede fundar o explicar la existencia de unidades reales, concebidas como intrínsecamente activas por Leibniz (AA VI, 4B: 1542/ OFC 2, 170). Por tal razón, como ya dijimos, necesita apelar a sustancias simples o mónadas que doten de orden y unidad a los seres vivos. «Es preciso que haya sustancias simples, puesto que las hay compuestas; pues lo compuesto no es sino un montón o aggregatum de simples» (GP VI: 607/OFC 2: 328). Estas sustancias simples, en tanto tales, es decir, sin partes, no son divisibles (GP VI: 607/OFC 2: 328), y no pueden constituirse por agregación ni tampoco pueden desagregarse en partes, por tanto, no pueden destruirse. De aquí que en la ontología leibniziana no haya nacimiento ni muerte naturales, es decir, por medios puramente mecánicos (Món. $\S 4$ y 5, GP VI: 607; GP IV: 480). Las sustancias simples o mónadas sólo pueden surgir por creación y ser destruidas por aniquilación. De esta manera, la unidad del ser vivo, su organización, que depende de estas unidades simples, no puede explicarse por medios puramente mecánicos, es decir, por mera agregación o yuxtaposición serial de partes, sino que ha de constituirse de una vez, de golpe, por así decir, por un acto de creación (GP VI: 544). Además de este argumento en favor de la creación explicitado por Leibniz, podemos proponer otro (no explicitado por Leibniz, al menos según nuestro conocimiento) que apela a la dimensión temporal. Si recordamos que la unidad del ser vivo no está constituida por una sola mónada, sino que, por un conjunto infinito de ellas, jerárquica y dinámicamente organizadas, se sigue de aquí que el ser vivo no podría constituirse de manera serial, como un proceso aditivo o de agregación, en un tiempo finito, pues esto implicaría una serie temporal infinita, que nunca terminaría, no constituyéndose por tanto la unidad o clausura funcional propia del ser vivo.

De esta manera, el ser vivo, en cuanto unidad real, supone una organización dada desde el comienzo, y su desarrollo es la transformación de esta unidad primigenia o preforma (ROBINET I: 41). Desde esta perspectiva, la mera indiferencia de las partes o componentes materiales no puede ser el fundamento de la organización de lo vivo. Por esto, Leibniz considera que la única repuesta coherente con su concepción del ser vivo es el preformismo (GP III: 368; VII: 565; Smith, 2011p. 165): «Estoy seguro que jamás un cuerpo orgánico de la naturaleza se forma a partir de un caos o de un cuerpo no orgánico» (GP VII: 
$562) ; «[\ldots]$ las leyes del mecanismo no pueden por sí solas formar un animal allí donde no hay aún nada organizado» (GP VI: 544/OFC 8: 515); «[...] los cuerpos orgánicos de la naturaleza jamás han sido productos de un caos o de una putrefacción, sino siempre de semillas en las que había, sin duda, una cierta preformación» (GP VI: 619/OFC 2: 339; ROBINET I: 41). En palabras de Duchesneau:

Leibniz excluye entonces la posibilidad de una epigénesis mecánica pura en la formación de los vivientes. Evoca para esto las metamorfosis de formas orgánicas observadas por Swammerdam, Malpighi y Leeuwenhoek: estas observaciones parecen justificar el hecho que podemos prescindir concebir el organismo como resultado de una aglomeración mecánica de partes, a las que convendría entonces acordar una suerte de principio material de animación (1998, p. 322).

Es decir, todo ser vivo proviene de otro ser vivo, o en otros términos: «toda organización proviene de una organización» (tal como resaltaba el biofísico Paul Weiss en su trabajo sobre el desarrollo embrionario (1968)). El desarrollo desde la perspectiva preformacionista consiste en un agrandamiento o despliegue de algo ya presente de alguna manera al comienzo, no existe epigénesis ${ }^{8}$. Afirma Leibniz: «[...] los cuerpos orgánicos en particular se desarrollan, como me parece, a partir de una suerte de preformación» (GP IV: 505). O bien: «Personas muy rigurosas en sus experiencias se han dado ya cuenta en nuestro tiempo de que se puede dudar de si alguna vez se produce un animal completamente nuevo, y de si los animales no están análogamente ya llenos de vida antes de la concepción en las simientes, y lo mismo para las plantas» (GP VI: 543/OFC: 514). De manera simétrica, la muerte para Leibniz consiste en un repliegue de los seres vivos, más allá de nuestra percepción consciente: «Hay sólo una posición razonable a tomar; y es aquella de la conservación no solamente del alma, sino también del animal y su máquina orgánica; aunque la destrucción de las partes más grandes lo haya reducido a una pequeñez que escapa a nuestros sentidos tanto como aquella en donde estaba antes de nacer» (GP IV: 480; GP VI: 553). Recordemos además, que en la ontología leibniziana incluso lo inanimado está lleno de vida, lleno de animales o seres vivos microscópicos. «Por mi parte, estimo que no es conforme ni al orden, ni a la belleza, ni a la razón de la creación, que solamente una pequeña porción de la materia esté dotada de un principio vital o de una actividad inmanente, puesto que la más grande

8 Quedan preguntas sin responder en esta propuesta, por ejemplo: ¿Por qué la preforma crece si ya está constituida? El crecimiento queda como algo extrínseco al proceso de desarrollo mismo. La preforma crecería hasta alcanzar un tamaño visible. ¿Existe alguna razón para establecer de antemano y como parte del proceso el tamaño que resulta adecuado? ¿Bajo qué condiciones se activa este crecimiento y por qué? 
perfección exige que la totalidad de la materia esté provista de esta manera» (GP IV: 512). Leibniz aúna los pliegues y repliegues de los vivientes en su desarrollo bajo la denominación de transformación o metamorfosis, siguiendo el modelo de las transformaciones de los insectos, puestas de relieve en esa época. «[Los animales] tan sólo se desenvuelven, se envuelven, se revisten, se desnudan, se transforman» (ROBINET I: 43/OFC: 347; GP IV: 480/OFC: 242).

Hoy podría parecer inadecuado concebir el desarrollo y el surgimiento de formas desde la perspectiva preformista, pero resulta una posición bastante coherente al considerar la concepción de la materia en el siglo XVII. Constituye una manera de dar cuenta de la insuficiencia del mecanicismo para explicar el surgimiento de unidades organizadas que se conserven en el tiempo, una manera de mostrar que los seres vivos, en cuanto organismos u organizaciones estructurales y dinámicas no se reducen a sus componentes materiales. Así, la apelación a un preformismo no resulta tan exótica ni extraña, una concepción arcaica propia de tiempos preevolucionistas, sino que parece una respuesta coherente ante una concepción demasiado pobre de la materia, y con ello, de los tipos de procesos que pueden darse en ella, o que pueden denominarse materiales. Y este es un problema que siguió presente en siglos posteriores hasta hoy en día, como encontramos por ejemplo en Bergson, quien en el contexto de la revolución industrial rechaza que el ser vivo sea comprendido como una suerte de fabricación, como un proceso en serie. Bergson considera que lo propio del ser vivo es la organización que implica una unidad no serial. ${ }^{9}$ Ideas similares las encontramos en el contexto de las teorías de autoorganización en las ciencias físicas y químicas de los años 1970. En este ámbito se plantean ideas que conducen a nociones tales como las de emergencia o autoorganización, el paso de un estado desordenado a otro ordenado $\mathrm{u}$ organizado debido a procesos de inestabilidad (Durán, 2013, p. 231). ${ }^{10}$

La segunda insuficiencia del mecanicismo se refería a la falta de una unidad organizacional propia, una unidad estructural y dinámica que establezca una diferencia no meramente fenoménica o puesta por quien percibe, entre el

9 Cf. Bergson (2007, p. 107). «[...] Bergson distingue entre organización y fabricación, adecuada contraposición para una época de industrialismo y producción en serie. La fabricación parte de elementos discretos y los ensambla o "asocia", en función de un plan predefinido, constituyendo una unidad, que no altera la identidad previa de estos elementos. [...] En la fabricación no encontramos nada que no esté puesto de antemano en la ejecución planificada. En la fabricación se aúnan los dos aspectos constitutivos de toda acción humana: planificación y ejecución, de los que derivan en última instancia el finalismo y el mecanicismo radicales. En cambio, en la organización de los seres vivos se trata más bien [...] de una unidad distribuida, de "disociación" más que de "asociación"» (Durán, 2013, p. 81).

10 Habría que estudiar con detalle si las teorías contemporáneas en física y química pueden enriquecer lo suficiente lo que entendemos por materia como para superar la brecha entre el ámbito de lo biológico y lo físico, sin caer en reduccionismos unilaterales (Durán, 2013). 
organismo o ser vivo y el medio ambiente; y que persista en el tiempo y en el espacio. Leibniz trae de vuelta las formas substanciales que el mecanicismo de corte cartesiano había dejado de lado (AA VI 4-B: 1542/OFC: 170). Complementa así el mecanicismo con la apelación a sustancias simples o mónadas. Estas sustancias dotan de unidad y continuidad a los cuerpos orgánicos; gracias a ellas los cuerpos orgánicos pueden ser una organización y una unidad estructural y dinámica en el tiempo: ser el mismo, sin ser lo mismo. «[...] las máquinas divinas [máquinas de la naturaleza] tiene la muy noble característica, que está más allá del alcance de lo que pueden lograr aquellas máquinas que somos capaces de inventar, de poder preservarse ellas mismas y producir copias de sí mismas» (Leibniz, 2016, p. 21).

Al concebir el ser vivo como un organismo, es decir, como unidad estructural y dinámica de complejidad infinita que persiste en el tiempo gracias a su mónada dominante, Leibniz puede explicar la conservación de la identidad del ser vivo en el tiempo y en el espacio, a pesar del continuo cambio de los materiales que lo constituyen y de las transformaciones radicales que sufre. Teniendo esto en cuenta, podemos entender cuando Leibniz nos dice que el animal cambia todo el tiempo permaneciendo sin embargo el mismo, sin ser lo mismo, en cuanto a su expresión fenoménica: «No hay más que una transformación de un mismo animal, según que los órganos son plegados diferentemente, y más o menos desarrollados» (GP IV: 481; Duchesneau, 2010, p. 97). La mera masa o cuerpo orgánico cambia todo el tiempo, pero las relaciones entre las mónadas permanecen, la organización permanece. En otras palabras, en la metafísica de Leibniz, el organismo permanece. La clausura o unidad de dominación o unidad o clausura funcional sería el fundamento de la unidad o clausura física o espacial. La unidad dinámica básica, el organismo o la organización, se mantiene en cada una de las transformaciones que experimenta el ser vivo, y que va más allá de los límites que desde nuestra perspectiva perceptiva asignamos al animal, a saber: su nacimiento y su muerte, que para Leibniz constituyen tan sólo desenvolvimiento y envolvimiento, respectivamente. Esta organización dinámica y estructural, este organismo, permite fundar la persistencia, conservación y estabilidad del ser vivo en el tiempo y el espacio. Leibniz, como parte de la tradición mecánica, describe esta persistencia en los Nuevos ensayos sobre el entendimiento humano, estableciendo una analogía con el funcionamiento del reloj:

En alemán se llama Unruhe, es decir, inquietud, al péndulo de un reloj de pared. Podemos decir que con nuestro cuerpo sucede igual, y que nunca podrá sentirse totalmente a gusto: pues en cuanto se sintiese así, cualquier impresión nueva de los objetos o un pequeño cambio en los órganos, vasos o vísceras, desequilibrará la balanza y le obligará a hacer un pequeño esfuerzo para volver a estar en el estado óptimo; se produce por 
tanto un combate perpetuo, que equivale, por así decirlo, a la inquietud del reloj, por lo cual dicha denominación es muy de mi agrado (GP V: 153; 1992, p. 190) ${ }^{11}$

Aquí Leibniz caracteriza la actividad del cuerpo como un equilibrio dinámico, es decir, un equilibrio que para mantener cierta condición debe estar en continua actividad de corrección, por así decir, para contrarrestar las perturbaciones y fluctuaciones que se producen de manera incesante. Este equilibrio dinámico, homeostasis en términos de la biología actual (Durán, 2013, p. 91), denominado por Leibniz inquietud, es considerado semejante al rol que cumple el péndulo en el reloj, que efectivamente mantiene la actividad del reloj dentro de cierto margen de equilibrio. Sin embargo, a pesar de la similitud destacada por el propio Leibniz, el ejemplo mismo pone de relieve la importante diferencia entre una máquina natural y otra artificial. Ciertamente, en ambos casos se produce un equilibrio dinámico, pero lo que se juega en ambos es completamente distinto. Un reloj sin péndulo no puede cumplir su función. Desde esta perspectiva el reloj pierde su funcionamiento, y en cierta medida su unidad funcional. Sin embargo, si esta pieza faltante es suplida el reloj recobra su funcionamiento. Vemos así que realmente el reloj no había perdido su unidad funcional, implícita en el diseño que coordina las partes, sino sólo una de las partes necesarias para funcionar. Diseño y funcionamiento (o actividad) no coinciden. En cambio, la pérdida de la capacidad de equilibrio dinámico por parte del ser vivo implica que el ser vivo pierde su unidad funcional. En el ser vivo diseño (organización) y dinámica van de la mano, por tanto la incapacidad de preservar el equilibrio dinámico, y por tanto la organización, implica la pérdida de la unidad funcional de manera radical. Mientras que en el reloj el equilibrio está asociado a una parte específica (el péndulo), en el ser vivo el equilibrio dinámico es una propiedad del conjunto, del todo y sus partes.

Con la incorporación de formas substanciales o mónadas y con ello la posibilidad de que un ser vivo pueda ser no un mero agregado de partes materiales de dificultosa distinción respecto a un ambiente y de casi milagrosa continuidad

11 Este texto es respuesta de Leibniz a lo dicho por John Locke en el Ensayo sobre el entendimiento humano: «5. La identidad de los animales. El caso no es tan diferente en los brutos, como para que cualquiera no pueda ver qué es lo que constituye un animal, y qué es lo que lo hace continuar siendo el mismo. Algo parecido tenemos en las máquinas, y pueden servir para ilustrar el caso. Por ejemplo, ¿qué es un reloj? Es llano que no es sino una organización o construcción de partes dispuestas adecuadamente para un cierto fin que sea capaz de ser realizado cuando se recibe el impulso de una fuerza suficiente. Si suponemos que esa máquina es un cuerpo continuo cuyas partes organizadas se reparan, aumentan o disminuyen por una constante adición o separación de partes no sensibles, en una vida común, tendremos algo muy semejante al cuerpo de un animal; pero con esta diferencia: que en un animal, la adecuación de la organización y el movimiento, en que la vida consiste, comienzan al mismo tiempo, pues el movimiento brota del interior; pero en las máquinas, como la fuerza procede del exterior, muchas veces está ausente cuando el órgano, sin embargo, está en orden y bien dispuesto para recibirla» (1999, p. 314). 
en el tiempo, Leibniz parece superar las insuficiencias del modelo del reloj como modelo del ser vivo. La organización ya no es una cuestión exterior al ser vivo, que surja de manera milagrosa a partir del choque puramente mecánico de las partes, sino que es parte constitutiva e intrínseca del ser vivo mismo. El ser vivo en Leibniz está organizado, es un organismo, por sí mismo. Leibniz complementa la concepción mecánica de los seres vivos, ejemplificada por el reloj mecánico, muestra las insuficiencias y limitaciones de este enfoque, pero sin dejar de lado su rol en las explicaciones del funcionamiento de los vivientes. Leibniz defiende el principio de que todo se hace mecánicamente en la naturaleza, pero no todo se puede explicar mecánicamente (Duchesneau, 1998, p. 330; GM III-2: 884). «Leibniz admite el principio según el cual todo se produce mecánicamente en el organismo viviente, pero no concedería que podamos explicar todo mecánicamente en las acciones que tienen lugar en él» (Duchesneau, 1998, p. 334).

\section{Conclusiones}

Nos hemos referido a dos insuficiencias del reloj como modelo del ser vivo, en el marco de la filosofía de la naturaleza del siglo XVII, el mecanicismo, a saber: el modelo del reloj deja sin explicar el origen de la estructura o la organización del ser vivo, y el reloj como modelo no establece un principio interno de actividad que fundamente la unidad dinámica y estructural del ser vivo, y que explique su actividad diferenciada y su conservación en el tiempo. Con su noción de máquina natural y de substancia corpórea o animal, Leibniz enfrenta estas insuficiencias del modelo puramente mecánico. En primer lugar, en su propuesta el ser vivo no se constituye de manera mecánica o serial, sino de una vez, es decir, por un acto de creación. Esta es la razón por la que apoya las teorías preformistas de la época y la inmortalidad de los seres vivos. Aunque estas afirmaciones puedan parecer anacrónicas hoy en día, apuntan a una cuestión que sigue siendo relevante, y que hoy se intenta volver inteligible con conceptos tales como emergencia o autoorganización, a saber, el origen de nuevas formas vivas, o de lo vivo mismo a partir de la materia. Leibniz rechaza el surgimiento de nuevas formas a partir de la materia, porque esto implicaría un proceso de duración infinita, debido a la infinita complejidad de los seres vivos. En segundo lugar, la conservación del ser vivo en el tiempo sólo es comprensible en la propuesta de Leibniz a partir de un principio de actividad intrínseco que lo dote de unidad, actividad y estructura a la vez. Plantea incluso en algunos pasajes ideas referidas a lo que hoy denominamos homeostasis. El ser vivo o animal constituye en Leibniz una estructura jerárquica y dinámica de mónadas, donde una de ellas constituye la mónada dominante que dota de 
unidad al ser vivo y organiza las mónadas subordinadas. La unidad del ser vivo en Leibniz es una unidad estructural y dinámica, una organización, que el filósofo alemán denomina «organismo».

La propuesta de Leibniz supera las deficiencias del modelo del ser vivo puramente mecánico, y está a medio camino entre un puro mecanicismo y un vitalismo dualista. Ciertamente la apelación a una creación primigenia no resulta una explicación suficiente a nuestros ojos. Por el contrario, vuelve imposible el dotar de un valor creativo al tiempo, o al desarrollo biológico mismo, pues en lo esencial las formas están dadas desde siempre. Las ideas evolutivas del siglo XIX apuntarán a dotar de novedad no sólo al comienzo del proceso sino al proceso mismo.

\section{Referencias}

ANDRAULT, R. "La raison des corps". Paris: VRIN, 2016. ARISTÓTELES. "Física". Barcelona: Gredos-RBA, 2007. BERGSON, H. "La evolución creadora". Buenos Aires: Cactus, 2007.

DESCARTES, R. "Euvres de Descartes publiées par C. Adam et P. Tannery". Paris: Léopold Cerf, 1909. [AT]

DESCARTES, R. "El tratado del hombre". Madrid: Ed. Nacional, 1980.

DESCARTES, R. "Los principios de la filosofía”. Barcelona: Alianza-RBA, 2002.

DESCARTES, R. "Discurso del método". Buenos Aires: Colihue, 2004.

DES CHENE, D. "Physiologia. Natural Philosophy in Late Aristotelian and Cartesian Thought". Ithaca: Cornel University Press, 1996.

DES CHENE, D. "Spirits \& Clocks. Machine \& Organism in Descartes". Ithaca: Cornell University Press, 2001.

DUCHESNEAU, F. "Les modèles du vivant de Descartes à Leibniz". Paris: VRIN, 1998. DUCHESNEAU, F. "Leibniz. Le vivant et l'organisme". Paris: VRIN, 2010.

DURÁN, R. "Autoorganización y estructuras disipativas: la imagen de naturaleza en Ilya Prigogine". Tesis doctoral inédita. Valparaíso, P. Universidad Católica de Valparaíso, 2013.

DURÁN, R. «Spontaneity, Body and Organism in Leibniz». (pp. 449-462). In: "Fürunserglückoder das glückanderer". Vörtrage des X. Internationalen LeibnizKongresses. Band II. Hildesheim: Georg Olms, 2016.

DURÁN, R. «Espontaneidad, cuerpo y organismo en Leibniz». (pp. 79-89). In: R. Casales y J. M. Castro (comps.). La modernidad en perspectiva. A trescientos años del fallecimiento de Leibniz. Granada: Comares, 2017.

FICHANT, M. «Leibniz et les machines de la nature». En: Studia Leibnitiana, Band XXXV/1, pp. 1-28, 2003.

JALABERT, J. "La théorie leibnizienne de la substance". Paris: PUF, 1947.

LEIBNIZ, G. W. "Sämtliche Schriften und Briefe". DeutschenAkademie der Wissenschaftenzu Berlin (ed.). Leipzig, Darmstadt, Berlin [AA], (1923-). 
LEIBNIZ, G. W. "Principes de la nature et de la grâce fondés en raison. Principes de la philosophie ou Monadologie". Publié intégralement d'après les manuscrits d'Hanovre, Vienne et Paris et présentés d'après des lettres inédites par A. Robinet. Paris: PUF, 1954. [ROBINET I].

LEIBNIZ, G. W. "Die philosophischen Schriften". 7 Vols. Ed. C. I. Gerhardt. New York: Georg Olms, 1978. [GP]

LEIBNIZ, G. W. "Nuevos ensayos sobre el conocimiento humano". Ed. J. Echeverría. Madrid: Alianza, 1992.

LEIBNIZ, G. W. 'Leibniz's 'New System' and Associated Contemporary Texts". Oxford, 1997. [WF]

LEIBNIZ, G. W. "Obras filosóficas y científicas”. 20 Vols. Granada: Comares, 2007-. [OFC]

LEIBNIZ, G. W. "The Leibniz-De Volder Correspondence, with Selections from Correspondence between Leibniz and Johann Bernoulli". Translated and edited by P. Lodge. New Haven: Yale University Press, 2013. [Lodge]

LEIBNIZ, G. W. “The Leibniz-Stahl Controversy”. Eds. F. Duchesneau; J. E. H. Smith. New Haven: Yale UniversityPress, 2016.

LOCKE, J. "Ensayo sobre el entendimiento humano". México D.F.: Fondo de Cultura Económica, 1999.

MATURANA, U.; VARELA, F. “De máquinas y seres vivos. Autopoiesis: la organización de lo vivo". Santiago de Chile: Universitaria, 1994.

MAYR, O. "Autoridad, libertad y maquinaria automática en la primera modernidad europea". Barcelona: Acantilado, 2012.

MONTAIGNE, M. de. "Journal de voyage de Michel de Montaigne en Italie, par la Suisse et l'Allemagne en 1580 et 1581 (Nouvelle édition)". Città di Castello: Lapi, 1889. PHEMISTER, P. "Leibniz and the Natural World. Activity, Passivity and Corporeal Substances in Leibniz's Philosophy". Dordrecht: Springer, 200.

RISKIN, J. "The Restless Clock: A History of the Centuries-Long Argument over What Makes Living Things Tick". University of Chicago Press, 2016.

ROSSI, P. "Los filósofos y las máquinas 1400-1700”. Barcelona: Labor, 1966.

SMITH, J. E. H., NACHTOMY, O. (eds.). "Machines of Nature and Corporeal Substances in Leibniz". New York: Springer, 2011.

SMITH, J. E. H. "Divine Machines. Leibniz and the Sciences of Life". Princeton: Princeton University Press, 2011.

WEISS, P. "Dynamics of Development". New York: Academic Press, 1968.

WILSON, C. "The Invisible World. Early Modern Philosophy and the Invention of the Microscope". Princeton: Princeton University Press, 1995.

WOOLHOUSE, R. S. "The Concept of Substance in Seventeenth Century Metaphysics". London: Routledge, 1993. 